\title{
"Il sottile veleno di tossiche fluorescenze": paesaggi urbani nella letteratura romena italografa tra emarginazione e soggettivazione
}

\section{"Il sottile veleno di tossiche fluorescenze": Urban Landscapes in the Literature of Romanian Diaspora, between Marginalization and "Subjectification"}

VALERIA Nicu [nicuvaleria3@gmail.com]

Università di Macerata, Italia

\begin{abstract}
RiAsSUNTO
Il presente intervento si propone di evidenziare all'interno della produzione letteraria romena italografa il rapporto disforico, alienante e spersonalizzante che il migrante instaura con il nuovo habitat, la nuova realtà. Vi si analizzano le valenze molteplici che si dispiegano in relazione allo spazio urbano: in particolar modo la ripresa delleredità simbolista nella riproposizione di topoi legati alla figura del poeta-clochard, le declinazioni contemporanee dell'uomo-merce e scarto nella società dei consumi, il migrante come "margine", soggetto dis-patriato e decentrato. Elementi narrativi costanti nella letteratura romena sono i due diversi timori che scandiscono la vita del migrante: il timore di cedere alla tentazione del richiamo verso le proprie origini attraverso l'idealizzazione del regime comunista, da un lato, e la paura di lasciarsi irretire dalle lusinghe ("le tossiche fluorescenze”) del consumismo nella nuova dimensione, dall'altro. Questi due sentimenti sono alla base delle strategie di appropriazione dello spazio e conseguente processo di soggettivazione personale.
\end{abstract}

\section{Parole Chiave}

Letteratura romena della migrazione; Italia; Città; Comunismo; Consumismo

\begin{abstract}
This paper aims to underline, in the context of the Italian literature of the Romanian diaspora, the alienated and impersonalized relationship that the emigrant establishes with the new habitat, the new reality. We can spot in it the various meanings blooming in the urban space: particularly the symbolist heritage of the topoi of the poet-clochard's character, the contemporary interpretation of the man-object, waste of the contemporary society, the emigrant as a "margin", as a person belonging to no country. Constant aspects of the Romanian literature's narrative are the two fears peculiar in the emigrant's life: the fear to yield to the nostalgic feeling towards his origins, through the idealization of the communist regime and, on the other hand, the fear to be bewitched by the newly experienced consumerism. These two predominant feelings are the origins of the strategy for the appropriation of the space and the consequent personal "subjectification".
\end{abstract}

\section{KEYWORDS}

Romanian Migration Literature; Italy; Urban space; Communism; Consumerism 
Nelle città straniere c’è una gioia sconosciuta,

la fredda felicità di un nuovo sguardo.

Gli intonaci gialli delle case [...] esistono

ma non per me. Non per me furono costruiti il municipio, il porto, il tribunale, la prigione. [...]

Le grandi bandiere degli alberi si agitano

al vento cosi come nei luoghi

a noi noti, e lo stesso piombo fu cucito

negli orli di lenzuola, di sogni,

dell'immaginazione folle e senza casa

Adam Zagajewski (2012: 101)

\section{Introduzione}

I flussi transnazionali attivati dai meccanismi della globalizzazione non solo tentano di tradurre l'altrove fisico in testo come succede nel genere odeporico (Cardona 2006: 295) ma, come ben evidenziato da Homi K. Bhabha nel suo volume I luoghi della cultura, contribuiscono a cambiare le coordinate della spazialità e della modernità:

la metropoli occidentale deve fare i conti con la sua storia postcoloniale, una storia narrata dal fluire dei migranti e rifugiati postbellici e che assume la forma di una narrazione indigena o nativa interna alla sua identità nazionale. [...] Queste culture [...] possono condizionare la modernità, introducendovi discontinuità e antagonismi e resistendo alle sue tecnologie oppressive e assimilazionistiche; ma possono anche sviluppare l'ibridità culturale insita nella loro condizione di frontiera, per 'tradurre' dunque ri-scrivere l'immaginario sociale sia della metropoli che della modernità. (2001: 18)

Questa "ermeneutica degli spazi urbani" si configura non solo come "risemantizzazione delle [...] topografie", ma soprattutto come ricodificazione delle soggettività, come problematizzazione del sistema culturale e ideologico di riferimento (Moll 2015: 168).

Come evidenziato da molti filosofi e pensatori, tutto il Novecento ha registrato l'appuntarsi dell'attenzione sulla questione dello spazio che s'impone sul tempo. Per Michel Foucault se "la grande ossessione che ha assillato il XIX secolo è stata [...] la Storia [...] quella contemporanea potrebbe invece essere considerata l'epoca dello spazio." (2011: 19). Bertrand Westphal invece, nel volume La Géocritique. Réel, fiction, espace teorizza una "spazializzazione del tempo":

On y verra comment les métaphores du temps tendent a se spatialiser depuis les lendemains de la Deuxième Guerre mondiale et de quelle façon l'espace a été revalorisé au détriment du temps qui, dans la critique et la théorie, avait exercé jusque-là une suprématie sans partage. Je m’attarderai ensuite sur une constante de l'espace contemporain: sa mobilité, qui est peut-être devenue chronique. $(2007: 17,45)$ 
La preminenza e la centralità della problematica spaziale assume nuovi significati nel contesto delle grandi diaspore migratorie e post-coloniali novecentesche laddove, a fronte di una mobilità cronica dell'individuo, lo spazio diventa un elemento determinante nel processo di generazione o dis-integrazione delle identità. Paola Zaccaria nel volume Mappe senza frontiere, si concentra proprio sul valore identitario dei luoghi: per lei la percezione spaziale è basata su una "relazione esperienziale che ciascuno ha con [un] luogo; è stratificata 'dentro la mente umana come un'insieme di immagini interiorizzate' e di conseguenza l'identità di un luogo collabora alla costruzione dell'identità personale" (1999: 56). Il luogo e la relazione che l'individuo vi istaura sono dunque elementi fondanti nel processo di Selbstbildung.

Con il presente intervento si intende affrontare la tematica del rapporto migrante-spazio urbano all'interno della produzione letteraria romena in italiano. Si prende in esame in particolare il rapporto disforico, alienante e spersonalizzante che il migrante instaura con il nuovo habitat, la nuova realtà. Vi si analizzano le valenze molteplici che si dispiegano in relazione all'ambiente urbano: in particolar modo la ripresa dell'eredità simbolista nella riproposizione di topoi legati alla figura del poeta-clochard, le declinazioni contemporanee dell'uomo-merce e scarto nella società dei consumi, il migrante come "margine", soggetto dispatriato e decentrato, consapevole però di proporsi con sguardo "altro" capace di plasmare la rappresentazione della realtà. Si esaminano i tentativi di regressione uterina al paesaggio materno della memoria, ma anche il doloroso approdo allo spazio violato e sconvolto della memoria che accentua l'impossibilità storica del nostos. Elementi narrativi costanti nella produzione letteraria rumena italografa sono i due diversi timori che scandiscono la vita del migrante: il timore di cedere alla tentazione del richiamo verso le proprie origini attraverso l'idealizzazione del regime comunista, da un lato, e la paura di lasciarsi irretire dalle lusinghe del consumismo ("le tossiche fluorescenze") nella nuova dimensione, dall'altro. Ne deriva un'oscillazione tra il rischio di abbandonarsi alla nostalgia e l'assoggettamento acritico al nuovo sistema. Forte è inoltre all'interno di questa produzione letteraria la sensazione che si stia assistendo a una chiusura delle città italiane verso il diverso: mentre la città come forma più avanzata della contemporaneità proteiforme dovrebbe essere un laboratorio di incubazione e di formazione dell'identità post-moderna, nomade e transculturale, negli autori romeni, invece, si denunciano le tendenze ad una provincializzazione mista ad un richiamo sempre più serrato al purismo etnico. Un fenomeno questo che è concomitante e conseguente al risveglio dei nazionalismi e dei regionalismi, all'acuirsi della febbre da possesso e controllo del territorio. Di fronte a una tale complessa e fragile realtà personale e contingente, gli autori romeni adottano strategie di resistenza e di appropriazione/colonizzazione dello spazio circostante dando così corpo a processi di soggettivazione personale che mirano, come più sopra evidenziato, a modificare le relazioni che incardinano l'individuo nello spazio della città e della contemporaneità.

\section{La casa}

Come evidenzia Stefano Manferlotti in La città degli immigrati. Spazio e memoria in Salman Rushdie e Hanif Kureishi, nella dislocazione, il soggetto transnazionale si spoglia del luogo di origine e: 
il divorzio fra la persona e i luoghi in cui si svolge la sua esistenza trova il suo immediato corrispondente analogico in una sorta di distonia tra anima e corpo. [Lo scollamento provoca] un generale ottundimento della sensibilità spaziale, e al limite, una vera e propria de-spazializzazione. [...] [Cosi] Nel momento in cui cade il rapporto sentimentale [...] fra soggetto umano e spazio, si inducono lo 'straniamento', la 'privazione', l'alienazione. (1995: 310)

Perciò le opere dei migranti intrecciano continuamente non-luoghi, non-case, non-persone. Sono teatro di "un'umanità instabile e in fuga, costretta a rinunciare al contatto fisso col territorio, al radicamento psicologico e sociale” (Pezzarossa 2010: 64). Un'umanità che vede spezzarsi il "corpus di immagini che forniscono ragioni o illusioni di stabilità" (Pezzarossa 2010: 62). Per Homi K. Bhabha ogni ridislocazione della propria casa nel mondo è connotata dall'estraneità al domestico, "l'unhomliness", “condizione di tutte le iniziazioni extra-territoriali e transculturali [che] [...] designa la fine di quel senso di appartenenza relativo a ciò che è homely, 'familiare', come le mura della propria abitazione" (2001: 22). "Costrette in una condizione transitoria e sospesa, - scrive ancora Fulvio Pezzarossa - per le figure prive dello statuto elementare della cittadinanza, si invertono i significati dell'esistenza: l'approdo a una dimora genera sensazioni opposte al senso di pace e sicurezza" (2010: 69). Analoghe considerazioni sono riferibili alle opere degli autori romeni concernenti la rappresentazione della casa e della città. Una prima analisi riguarda, infatti, l'estraneazione dell'individuo sradicato a contatto con la nuova realtà, estraneazione dovuta al fatto che il cambiamento dell'habitat umano porta ad una diversa percezione del tempo e dello spazio. I testi tradiscono l'impossibilità di aderire ad un ambiente estraneo, privo di ricordi, che veicola un costante senso di frustrazione, di disorientamento e negato autoriconoscimento che si dispiega a partire dall'ambiente domestico. Scrive così Irina Turcanu nel racconto Oltre la paura, legato all'esperienza autobiografica della migrazione:

Nina si rifugiò nella sua stanza tetra, senza ricordi e tracce di sé. Un letto, una scrivania, un armadio: i mobili di un albergo, di una prigione arredata più dell'indispensabile. (2011: 248)

Allo stesso modo Eliza Macadan, evocando la sua vita pellegrinante a seguito degli impegni giornalistici, delinea la casa come una sorta di appendice commerciale, un aguzzino freddo e impietoso in un "Occidente, solo disposto alle relazioni economiche che reificano l'esistenza altrui” (Pezzarossa 2010: 68):
Non c’è casa
non cercarla più
c’è solo
un affitto costoso
su una terra padrona.
(Macadan 2012: 14)

La casa, come ci ricorda Remo Bodei ne La vita delle cose, è il guscio dell'intimità: l'ambiente, gli oggetti, i luoghi incorporano i ricordi, le aspettative, i sentimenti, diventando dirette "appendici” dell'io e simboli che tengono insieme le schegge disperse di una vita (2009: 50). Le autrici 
citate, al contrario, ci dicono di muri privi di memorie e di identità, ci raccontano di un vuoto mai risemantizzato, di un universo che non ha nulla di familiare (heimlich) e che irrompe, invece, in tutta la sua forza straniante. La casa si configura, infatti, di volta in volta come un luogo neutro, quasi un albergo, uno spazio occasionale e provvisorio oppure come una prigione, uno spazio opprimente e costrittivo che genera nel soggetto un rapporto disforico.

Viene così a cadere il senso della casa come involucro primo della personalità, il bachelardiano nido di cui si riveste il nostro corpo in funzione protettiva nei confronti delle aggressività esterne. Essa diventa ambiente respingente e inospitale nel quale è impossibile riconoscersi, trovarvi requie. Ne deriva un senso persistente di instabilità e di non-appartenenza, acuita dalla freddezza e dall'impersonalità della città.

C’è tuttavia in alcuni testi una volontà di resistere e di "colonizzare" lo spazio a partire proprio dalla regolamentazione del perimetro domestico. Così ironicamente Mihai M. Butcovan, in Allunaggio di un immigrato innamorato, elenca fin da subito le regole per gli ipotetici avventori del proprio micro-appartamento milanese pur non possedendo ancora il mobilio:

Mi prese poi il raptus dellordine della stanza. [...] Sono due metri per quattro più il bagno dueperuno.

Col pirografo ho scritto l'entrée:

"Voi c'entrate in questa casa... uscendo lasciate qualche speranza?

"Ammonimento: qui l'arredo è stato acquistato a millelireprepezzo. Siete pregati dunque di contribuire alla manutenzione di tutti i mobili di cui farete uso.

"Consigli: gli accendini sono sul tavolo. servitevi pure! Sono legati con la cordicella, come alle poste, e segnalati da apposite bandierine rosse importate dalla Russia, che vi indicano la posizione nonché il divieto di asporto. [...]

Questo benvenuto l'ho appeso sull'anta interna dell'armadio. Rimarrà lì fino a quando non ci saranno il divano, gli accendini e ... la casa. (2006: 96-97)

Nel brano citato emerge non solo la consapevolezza che "nell'abitazione si proiettano le relazioni della socialità, attraverso il processo culturale che consente l'affermarsi del senso di appartenenza, trasformando un ambiente (place) nello spessore di uno spazio vivo (space)" (Pezzarossa 2010: 60), ma anche la consapevolezza che "lo spazio intimo della casa' [...] è un luogo che incardina relazioni di potere" (Pezzarossa 2010: 76). Già bell hooks in Homeplace a site of resistence, nell'ambito dell'analisi della lotta per la liberazione dei neri e in particolare delle donne nere, notava il ruolo dello spazio domestico come luogo di resistenza alle pressioni esterne che plasmano e manipolano l'individuo minoritario e subalterno e anche come luogo dal valore sovversivo, rifugio in cui rigenerarsi e riorganizzarsi psicologicamente e politicamente:

$[\ldots]$ one's homeplace was the one site where one could freely confront the issue of humanization, where one could resist [...] where we could restore to ourselves the dignity denied us on the outside in the public world. (1990: 42)

È chiaro in Butcovan il tentativo di ritagliarsi lo spazio per creare un homeplace, quanto meno nell'immaginario, un proprio nido-fortilizio per la difesa del proprio microcosmo, ma 
anche il desiderio di intessere relazioni con l'alterità - "a meaninful community of resistence" (Hooks 1990: 47) - a partire dalla necessità di creare regole condivise, la volontà di non lasciarsi colonizzare dallo sguardo dell'altro, nonché il profondo bisogno di territorializzare/colonizzare il macrocosmo a partire dal proprio microcosmo.

\section{La città}

Gli scrittori rumeni, rifacendosi ai topoi già ottocenteschi della città quale simbolo di degrado $\mathrm{e}$ di emarginazione sociale, dipingono la città post-moderna come luogo di esclusione abitato da profonde ingiustizie sociali dove il discrimine si palesa nelle più svariate forme.

Macadan, per esempio, descrive la città quale organismo aberrante che vomita i suoi abitanti, una volta giunta a saturazione:
preferisco le foglie
sul cielo
buttate in su
dall'altezza degli alberi
allombra delle mura
in una metropoli
che mi abortisce
ogni giorno
(Macadan 2012: 41)

Autori come Mihai Mircea Butcovan (2010) e Viorel Boldis (2006), invece, riattualizzano il topos del diseredato, del barbone, del senza-casa. Tema questo dell'homeless e dell'outsider che dispiega una pluralità di valenze:

\section{Senzatetto}

Dove andare

Steso

Non lacrimare

Peso

Di una vita senza nome

Senza dove e senza come

Errabondo

Nel mondo

E nel buio più profondo

Io mi nascondo.

(Butcovan 2010: 92) 


\section{Navigli}

[...]/Straniero dormo sui giornali e sui Navigli.

(Butcovan 2010: 83)

\section{Qualche volta}

Qualche volta sono quello

che gli altri scartano,

immondizia del mondo,

della società. [...]

(Boldis 2007: 8)

Gli estratti citati testimoniano una triplice alienazione del migrante nello scenario metropolitano: l'alienazione dello straniero sradicato, continuamente fuori posto, quella del poetaclochard e, infine, il dramma dell'uomo-merce e, quindi, possibile scarto nella società dei consumi.

E chiaro che in questi autori la figura del reietto, in parte riconducibile al topos romantico e simbolista della marginalizzazione cronica del poeta-straniero, serve a marcare, da un lato, l'esclusione dalla società, con tutta la sofferenza che ne deriva, dall'altro, l'acquisizione orgogliosa, proprio in virtù della marginalità, di un doppio sguardo, capace di proporre rappresentazioni alternative della realtà in cui il migrante si inserisce. Sicuramente, come evidenzia Chiara Mengozzi, nell'articolo Città e modernità: nuovi scenari urbani nell'immaginario della "letteratura italiana della migrazione", lo sguardo eccentrico e dislocato del migrante funziona come meccanismo di straniamento rispetto agli spazi urbani di arrivo (2008: 3). È interessante, a questo proposito, soffermarsi sulla riattualizzazione della figura del reietto urbano. Gli scrittori, ricorrendo a questa figura letteraria, non intendono soltanto denunciare la sensazione di costante isolamento dal consesso umano. Desiderano piuttosto sottrarsi, attraverso il gesto della scrittura, alla violenza epistemologica dello sguardo autoctono, dando vita a processi di empowerment, per esempio tentando di inserirsi all'interno della tradizione letteraria del paese di arrivo attraverso la pratica dell'intertestualità e del citazionismo, che indiscutibilmente conferiscono prestigio al "nuovo" scrittore. Si tratta perciò, a ben vedere, non tanto o non soltanto di denuncia sociale, quanto piuttosto di un dispositivo retorico volto alla ricerca di legittimazione e all'avviamento di processi di soggettivazione. La stessa chiave di lettura può essere utilizzata anche per interpretare alcune poesie, come Da solo nella fossa comune di Boldis:

\section{Da solo nella fossa comune}

Da solo nella fossa comune ci sto

Nessuno da questo è immune, però, [...]

Comunque, ammettendo alcune lacune,

Non so, se ci sia qualcuno

Con me nella fossa comune. (2006: 1)

Qui l'autore si richiama all'isolamento denso di rifiuti e solitudine del migrante, ma nello stesso tempo, attraverso il riferimento all'immagine della fossa comune dei genocidi novecenteschi, 
radicalizza e declina in chiave contemporanea il tema baudelairiano dell'immersione nella folla anonima, un'immersione che diventa una solitudine-morte dell'individuo proiettato nei grandi e incontrollabili scenari metropolitani.

L'esclusione sociale e il discrimine a volte diventa invisibilità nelle opere dei romeni italografi. Pochi luoghi come la metropoli gettano l'individuo nello sconforto dell'anonimato. Un "anonimato architettonico", come lo definisce Manferlotti (1995), che si accompagna "all'anonimato esistenziale" e che può evolversi fino a condurre alla totale spersonalizzazione e reificazione dell'io, come nella poesia di Boldis, Come una statua:

\author{
Essere straniero \\ è peggio che essere una statua. \\ Stai lì inerte, affamato e infreddolito[...] \\ Gli altri nemmeno ti odiano, \\ ti guardano appena annoiati, \\ si stringono il naso \\ e si fanno i fatti loro.
}

(2006: 27)

Nello scenario urbano l'individuo migrante assume di volta in volta i connotati del barbone, dell'invisibile o dell'inviso alla società quando non elemento neutro, indifferente al transito e all'affaccendarsi quotidiano degli autoctoni. In quanto figura sociale debole, il migrante può risvegliare nei passanti lo spettro della marginalità e della povertà e di conseguenza mettere in moto il meccanismo di difesa dell'impassibilità.

Alla tematica dell'invisibilità è connesso un altro nucleo problematico molto presente all'interno della produzione italografa rumena: la conflittualità sociale legata al dominio del territorio e alle chiusure ideologiche nazionaliste/regionaliste. In molte opere recenti lo scenario urbano si configura come un campo di lotta per la territorializzazione, da intendersi come reazione locale dell'invasione del globale. Come spiega, infatti, Giampaolo Nuvolati in Lo sguardo vagabondo. Il flâneur e la città da Baudlaire ai postmoderni:

[le] ingenti masse di popolazione che tendono a concentrarsi nella città, [tendono] a integrarsi, ma anche a confrontarsi aspramente tra loro e con le popolazioni residenti per l'accesso ai servizi, l'occupazione dello spazio e l'uso delle risorse, a proporre modelli culturali spesso contrastanti con i sistemi dei valori locali. (2006: 7)

Così ad esempio, nel racconto Due dita di troppo, Butcovan denuncia come, a Milano, il parco che il protagonista attraversava quotidianamente delineando personalissime mappe emotive, si svuota all'improvviso per la collocazione all'ingresso dei dissuasori mobili che, su richiesta dei cittadini, devono "porre fine alle feste abusive, chiassose e rissose, dei sudamericani" (2014: 12). Il migrante - sembra suggerire Butcovan - rischia di essere sentito come soggetto inopportuno, in quanto provoca imbarazzo e fastidio negli "autoctoni" a causa di una diversa fruizione dello spazio. Egli traccia l'immagine di una città che da spazio urbano misto, ricco di suggestioni e vissuti, viene progressivamente stretto nella morsa di una politica securitaria e denuncia, inol- 
tre, i meccanismi di manipolazione massmediatica dellopinione pubblica. Tutta la scrittura di Butcovan, in effetti, è venata dell'inquieto avanzare dei movimenti padani, delle paramilizie, (“Diamo il parco ai cittadini, non regaliamolo ai rom” (2014: 10)), aspetto puntualmente ridicolizzato dall'autore. Leggiamo infatti nell' Allunaggio di un immigrato innamorato:

La cena è stata squisita. [...]

Ho capito presto che non sapevano fossi rumeno. E se non l'hanno capito dalla pronuncia vuol dire che ho maneggiato bene le forchette. All'italiana. Io non mi sento quando parlo. Sì, lo so che non va bene, ma i tuoi hanno escluso che potessi essere "da Roma in giù" e hanno detto molte cose sui terroni e sugli extracomunitari. Non ebbi il coraggio di difendere le rispettive categorie. [...] In sintesi: cena padana nella Grande Famiglia Padana, madre padana, padre padano, figlia padana, [...] zio padano [...] nonno padano e nonna padana.

"Papà padano, esco con la mia fidanzata padana, mi dai le chiavi padane della macchina padana e la mancia padana?"

"Metti il preservativo padano, che fa freddo!" (2006: 63)

La stessa tematica attanaglia anche Ingrid Beatrice Coman. Nel romanzo Dodici più un angelo, in un'indefinita città del Nord, si degenera nello scontro di civiltà, nella guerriglia urbana: il "clan dei puri" scade nel fanatismo violento contro migranti e barboni. Esso viene alimentato e supportato dalla propaganda politica demagogica e populista: si evoca la nostalgia di un'immagine domestica e familiare di un momento "ideale" prima dell'arrivo degli immigrati in cui in città si sentiva parlare soltanto in italiano e non vi erano barboni, né criminalità. Una propaganda non soltanto violenta, ma anche antistorica, poiché finalizzata, tra le altre cose, a far cadere nell'oblio la repulsione per la migrazione italiana interna dei decenni precedenti. Anche nel testo della Coman, dunque, si mettono a nudo i dispositivi di mistificazione che portano alla creazione di una "cultura nazionale omogenea" (Bhabha 2001: 16), tramite l'esclusione delle diversità e la costruzione, in tutte le epoche, di nemici interni ed esterni, di un "loro" da opporre al "noi" (Eco 2011: 9-36)

\section{Ideologie a confronto}

Ciò che più contraddistingue la letteratura dei romeni italografi è la dicotomia Oriente/Occidente, Comunismo/Consumismo. Gli autori sono divisi tra il trauma storico del Comunismo di Ceausescu (legato al loro percorso biografico) e il Neoliberismo, il Capitalismo, il Consumismo occidentale. Anche la rappresentazione della città viene condizionata da questa dicotomia: sia la città "occidentale" italiana che la città "orientale" romena alternano valenze entusiaste legate alla cultura pop materialista a valenze negative: diventa una giungla del capitale senza regole che plasma uno spazio patologico.

La scrittrice Irina Turcanu ai suoi esordi, nel romanzo Alia, su un sentiero diverso, vagheggia il paese natale al quale era ancorata la sua memoria cristallizzata. Lo descrive infatti quale luogo idilliaco rurale in cui si conservano le tradizioni e i valori dell'umanità e della solidarietà contrariamente alle feste commerciali italiane/occidentali (2008: 50). Nel romanzo La pipa, Mr. Ceb 
e l'Altra, al contrario, la protagonista si muove nel mondo consumistico e metropolitano milanese, tra feste in discoteca e abiti alla moda che richiamano alla mente le immagini delle serie televisive americane in stile Sex and the city. In questo secondo romanzo, la scrittrice ricorre massicciamente all'immaginario della cultura pop metropolitana come gioco sociale della riconoscibilità, come strategia di integrazione e di affermazione. Nel suo quarto romanzo, Rigor Artis, ambientato a Bucarest, viene a cadere il velo di memoria blanda che avvolgeva il paese natale, "il feticismo delle identità pietrificate" tipica della "riscoperta delle proprie tradizioni culturali indigene" dei popoli subalterni (Bhabha 2001: 21). Così la città diventa ricettacolo di degrado umano in seguito alla caduta del Comunismo e all'ingresso incontrollato dei modelli capitalistici occidentali.

In generale questi scrittori dimostrano di essere scissi tra il rischio di revisionismo nostalgico e la demonizzazione del comunismo, mentre tentano di evitare l'assoggettamento al neoliberismo. Questa lacerazione diventa ancora più profonda nell'ambiente metropolitano ${ }^{1}$ dove le contraddizioni del capitalismo si manifestano in maniera più ostentata: la promessa di libertà e di pluralismo deve qui fare i conti con un più subdolo assoggettamento dell'individuo al capitale. Si può dire che la loro presa di coscienza sia assimilabile a quella che in tempi recenti ha toccato tutti i paesi dell'ex blocco sovietico ${ }^{2}$, la stessa che ha coinvolto la Germania dell'Est, che nella riunificazione del paese denuncia un nuovo colonialismo ${ }^{3}$. Come per i tedeschi della ex DDR, sopiti gli entusiasmi, anche i romeni avvertono che l'arrivo del capitalismo non sia stata una mera liberazione. Per giunta le promesse di libertà e di riscatto economico vagheggiate al momento dell'ingresso in Europa sono state ampiamente disattese ${ }^{4}$. Le dinamiche di sfruttamento e di dominio economico interne a certe aree dell'Europa sono state ben evidenziate nello studio di Lynda Dematteo, La corsa verso la Romania degli imprenditori italiani, condotto nell'ambito del progetto Fabbriche d'Europa, in cui si prendono in analisi i risvolti del capitalismo selvaggio in Europa:

Prima ancora della caduta del Muro di Berlino, i piccoli imprenditori italiani hanno cominciato a delocalizzare le loro unità di produzione nella Romania comunista per meglio resistere alla concorrenza dei paesi emergenti. Gli imprenditori italiani chiamano oggi Far East i vecchi paesi del Blocco orientale, che sono passati in meno di dieci anni, dal 'socialismo reale' al 'capitalismo reale, poiché si tratta per loro di una nuova Frontiera nel cuore dell'Europa. [...]

Se l'Europa istituzionale è ancor oggi in panne, l'integrazione europea non cessa di realizzarsi, ma non necessariamente come ci si aspettava, bensì secondo delle configurazioni informali e talvolta anche illegali. Studiando dunque questi spazi transfrontalieri dove si scambiano non

1 Cfr. il concetto di "postcommunist carnivalesque"della pop culture in Fragmented identities. Popular culture, sex, and everyday life in Postcommunist Romania di Denise Roman (2003: 47-48).

2 Sulla questione delle difficoltà e contraddizioni delle democrazie post-comuniste e del passaggio all'economia di mercato cfr. Rossen Vassilev (2011); Durandin, (2000); Di Quirico (2010); Dallara (2010).

3 Cfr. la Ostalgie in Matthias Frings (2012); l'Anschluss, annessione in Vladimiro Giacché (2013); Bruni de la Motte (2009).

4 I paesi dell'Est Europa come la Romania e la Bulgaria hanno presto compreso di far parte di un'Europa di secondo ordine: "A new curtain is falling across eastern Europe dividing north from south, west from east, rich from poor [...]. As Hungary, Poland, and the Czech Republic sprint into a future of democracy and market economies, Romania and Bulgaria slide into Balkan backwardness and second-class citizenship in the new Europe" (Longwoth 1994). 
soltanto delle merci, dei capitali, dei savoir-faire, ma anche dei corpi (penso in particolare alla prostituzione), spero di cogliere i processi di decostruzione degli spazi di appartenenza nazionali - ed esplorare le nuove fabbriche d'Europa. (Dematteo 2009: 1, 14, 36)

Il tema della mercificazione e della disumanizzazione attraversa gran parte della produzione letteraria degli scrittori romeni italofoni. La loro riflessione si appunta spesso sulle deleterie conseguenze delle nuove forme di schiavitù che si palesano in particolare nella mercificazione dei corpi, come in Ecatombe di Butcovan:

\author{
[...] farfalle pubblicitarie \\ di negozi falliti \\ poveri in saldo \\ colf alla mano \\ e $3 \mathrm{X} 2$ nelle confessioni pasquali. \\ (2010, p. 48)
}

La loro riflessione si spinge fino a toccare temi delicati come quello dei contingenti di donne che, abbandonando le proprie famiglie, ricadono nell'ambito dello sfruttamento domestico e sessuale, nel lavoro di cura e nella tratta della prostituzione. Il racconto Pegno per mamme di Ingrid B. Coman rende questi drammi attraverso lo sguardo sensibile e straniato di un bambino, un orfano bianco, che tramite la metafora del pegno denuncia l'atrocità dei meccanismi globali. Così la madre protagonista del racconto diventa un pegno in cambio del quale si ottiene "il miglioramento" della vita:

[...] avevo colto, qua e là, masticati in sordina agli angoli della casa, discorsi su sacrifici e bisogni e una vita migliore e soldi [...] ho intuito che [...] quel paese lontano e impietoso mi portava via la mamma e ci dava, ogni tanto, soldi in cambio e continuavo a chiedermi chi avrà mai fatto quel baratto mostruoso [...].

Solo dopo che te ne sei andata mi sono accorto che esistono banchi del pegno anche per mamme. Ti immaginavo dimenticata su uno scaffale chissà dove, $[. .$.$] mentre un altro Costache, altrettan-$ to brutto e avido, contava, nella sua lingua straniera, quanti soldi valevi. (2012: 14-15)

Anche la scrittrice Claudia Livia Bazu nel suo racconto Arterie (2006), attraverso lo sguardo di una ragazza dell'Est che lavora in un night club, porta alla luce la logica della violenza e del profitto della società post-consumista che nega l'individuo spingendolo in un vortice di illusione e di piacere. Così anche nella poesia Autobiografie (2003) il "sottile veleno nelle tossiche fluorescenze" allude al rischio di lasciarsi catturare e irretire dalle luci dell'opulento Occidente:

Gridai e piansi sbattendo la porta

dietro cui imparai a essere straniera

per l'ultima volta e via.

Una stazione affollata,

orari e biglietti, turisti, negozi e luci al neon. 
Nessuno mi accolse, nessuno mi disse

che c'era un sottile veleno

nelle tossiche fluorescenze che respirai.

Corpi modellati e sorrisi truccati

camminavano in sgargianti sudari

in perfetta finzione di umanità. [...]

Una prigione di parole che non/ significava mai me

e tutto era uno scadente surrogato di tutto [...].

(Bazu 2003: 67)

\section{Conclusioni}

Gli scrittori e le scrittrici trattati in queste pagine, di fronte alla giungla umana e urbana dei paesi di arrivo e, talvolta, di origine, in alcuni casi si abbandonano alla sconforto, in altri reagiscono con ironia, più spesso tentano di resistere, mettendo in atto diverse strategie di appropriazione dello spazio. Emblematico a questo proposito il racconto di Butcovan, Due dita di troppo, dove il protagonista, alla maniera del barboncino che è solito incontrare durante le sue passeggiate, decide di orinare nel parco - chiuso ai migranti - per segnare il territorio e impossessarsi dello spazio: un vero e proprio gesto di appropriamento sovversivo con valenza di pratica di insubordinazione civile. Tentativi di radicamento sono anche gli interventi sulla lingua italiana: Butcovan nella sua produzione letteraria ricorre spesso al miscuglio pirotecnico, ludico e parodico del linguaggio per uscire da una logica meramente legata al territorio e proiettarsi su una dimensione di comunità psicologica e sovra territoriale che si definisce soprattutto su base linguistica. Molta critica ha già evidenziato "la potenzialità identitaria e di cittadinanza implicita nell'uso letterario della lingua. [...] La scelta dell'italiano come mezzo di espressione artistica costituisce [...] il primo segnale di appartenenza e la più esplicita richiesta di cittadinanza" (Fracassa 2012: 74-75). Come spiega ancora Fracassa "la proprietà del linguaggio", il citazionismo, il ricorso e il sovvertimento dei generi della tradizione letteraria non solo italiana, ma anche europea, mira a "ridiscutere, in letteratura, ciò che è dentro e ciò che è fuori, dal canone, dalla storia letteraria, dai confini, geografici e mentali” (2012: 75). In definitiva la strategia prima e più radicale è il ricorso stesso alla scrittura che assume valore "abitativo": nella giostra delle dis-appartenenze che connotano l'esperienza diasporica, in assenza di una casapatria, abortiti dallo spazio urbano, gli scrittori migranti spazializzano la parola, adottano come propria dimora la scrittura. Quest'ultima, come sottolinea Said (2007: 139), per quanto fragile e vulnerabile, diventa l'unica casa davvero disponibile a partire dalla quale ricrearsi un'identità e relazionarsi con gli spazi della modernità. 


\section{Riferimenti bibliografici}

Barbarulli, C. (2010). “Era quella bellezza della sua città polifonica, sussurrante...”: abitare lo spazio urbano in scrittrici fra lingue e culture. In: C. Barbarulli (Ed.), Scrittrici migranti: la lingua, il caos, una stessa (pp. 125-146). Pisa: ETS.

Bazu, L. C. (2003). Autobiografia. In: Impronte. Scritture dal mondo (pp. 67-68). Nardò: Besa.

Bhabha, H. K. (2001). I luoghi della cultura. Roma: Meltemi.

Bodei, R. (2009). La vita delle cose. Roma-Bari: Laterza.

Boldis, V. (2006). Da solo nella fossa comune. Bologna: Gedit.

Butcovan, M. M. (2006). Allunaggio di un immigrato innamorato. Nardò: Besa.

. (2014). Due dita di troppo. In: G. Kuruvilla (Ed.), Milano d’autore (pp. 7-14). Milano: Morellini.

Butcovan, M. M., \& Belli, M. (2010). Dal comunismo al consumismo: fotosafari poetico esistenziale romenoitaliano. Ferrara: Linea BN Edizioni.

Cardona, G. R. (2006). I viaggi e le scoperte. In: I linguaggi del sapere. Roma-Bari: Laterza.

Coman, I. B. (2012a). Dodici più un angelo: un insolito straniero, una città da salvare e la matematica nascosta delle anime. Murazzano: Ellin Selae.

. (2012b). Satul fara mamici. Il villaggio senza madri. Milano: Rediviva Edizioni.

Dallara, C. (2010). La Romania in Europa: adesione senza integrazione? La criticità del settore giudiziario e anti-corruzione. Rivista italiana di scienza politica, 1, 83-112.

De La Motte, B. (2009). East Germans lost much in 1989. The Guardian [online], 8 Novembre. In: http:// www.theguardian.com/commentisfree/2009/nov/08/1989-berlin-wall.

Dematteo, L. (2009). La Corsa verso la Romania degli imprenditori italiani. Circolazione, assimetrie e narrazioni. [online]. In: http://www.institutdelors.eu/media/etud69-ldematteo-it.pdf?pdf=ok.

Di Quirico, R. (2010). La democrazia si è fermata a Bucarest. Limiti e prospettive del processo di democratizzazione nei paesi ex-sovietici nuovi vicini dell'Unione europea. Rivista italiana di scienza politica, 1, 59-82.

Durandin, C. (2000). La Romania al bivio tra nostalgie nazionaliste e miraggi europei. Limes - Rivista di geopolitica, 3, 182-192.

Eco, U. (2011). Costruire il nemico e altri scritti occasionali. Milano: Bompiani.

Foucault, M. (2011). Spazi altri. I luoghi delle eterotopie. Milano-Udine: Mimesis Edizioni.

Fracassa, U. (2012). Patria e lettere. Per una critica della letteratura postcoloniale in Italia. Roma: Giulio Perrone Editore.

Frings, M. (2012). L'ultimo comunista. Roma: Voland.

Giacché, V. (2013). Anschluss: l’unificazione della Germania e il futuro dell'Europa / Vladimiro Giacché. Reggio Emilia: Imprimatur.

Hooks, B. (1990). Race, Gender, and Cultural Politics. Boston, MA: South End Press.

Lombardi-Diop, C., \& Romeo, C. (2012). Postcolonial Italy. Challenging National Homogeneity. New York: Palgrave Macmillan.

Longworth, R. (1994). Bulgaria, Romania Resist Pull of the West. Chicago Tribune [online], 10 Ottobre. In: http://articles.chicagotribune.com/1994-10-10/news/9410120048_1_romania-and-bulgaria-bulgarian-president-zhelyu-zhelev-czech-republic.

Macadan, E. (2012). Paradiso riassunto. Novi Ligure: Joker. 
Manferlotti, S. (1995). La città degli immigrati. Spazio e memoria in Salman Rushdie e Hanif Kureishi. In: C. Pagetti (Ed.), La città senza confini. Studi su’immaginario urbano nella letteratura di lingua inglese (pp. 301-311). Roma: Bulzoni Editore.

Mengozzi, C. (2013). Narrazioni contese. Vent'anni di scritture italiane della migrazione. Roma: Carocci.

Mengozzi, C. (2008). Città e modernità: nuovi scenari urbani nell'immaginario della "letteratura italiana della migrazione”. In: C. Gurreri, A. M. Jacopino, \& A. Quondam (Eds.), Moderno e modernità: la letteratura italiana [online]. Roma: La Sapienza Editore. In: http://www.italianisti.it/FileServices/Mengozzi\%20Chiara.pdf.

Nuvolati, G. (2006). Lo sguardo vagabondo. Il flâneur e la città da Baudlaire ai postmoderni. Bologna: Il Mulino.

Papotti, D. (2011). L'approccio della geografia alla letteratura della immigrazione. Riflessioni su alcune potenziali direzioni di ricerca. In: F. Pezzarossa, \& I. Rossini (Eds.), Vent'anni di scritture della migrazione in Italia (pp. 65-84). Bologna: CLUEB.

Pezzarossa, F. (2010). Una casa tutta per sé. Generazioni migranti e spazi abitativi. In: L. Quaquarelli (Ed.), Certi confini. Sulla letteratura italiana della migrazione (pp. 59-84). Milano: Morellini Editore.

Righini, M. (2003). Città degli incubi. In: G. M. Anselmi, \& G. Ruozzi (Eds.), Luoghi della letteratura italiana (pp.142-152). Milano: Mondadori.

Roman, D. (2003). Fragmented Identities. Popular Culture, Sex, and Everyday Life in Postcommunist Romania. Lanham: Lexington Books.

Said, E. W. (2007). Riflessioni sull'esilio. Scritture migranti, 1, 127-141.

Todorova, M. (2009). Imagining the Balkans. New York: Oxford University Press.

Turcanu, I. (2008). Alia, su un sentiero diverso. Torino: Seneca.

- (2010). La pipa, Mr. Ceb e l'Altra. Padova: Ciesse Edizioni.

_. (2011). Oltre la paura. In: D. Finocchi (Ed.), Lingua Madre Duemilaundici. Racconti di donne straniere in Italia. Torino: Edizioni SEB 27.

. (2015). Rigor Artis. Roma: Absolutly Free Edition.

Vassilev, R. (2011). The Tragic Failure of "Post-Communism" in Eastern Europe [online], 8 marzo. In: http:// www.globalresearch.ca/the-tragic-failure-of-post-communism-in-eastern-europe/23616.

Westphal, B. (2007). La Géocritique. Réel, fiction, espace. Paris: Les Éditions de Minuit.

Zaccaria, P. (1999). Mappe senza frontiere. Cartografie letterarie dal Modernismo al Transnazionalismo. Bari: Palomar Edizioni.

Zagajewski, A. (2012). Dalla vita degli oggetti: poesie 1983-2005. Milano: Adelphi. 